\title{
Chatbots and New Audience Opportunities for Museums and Heritage Organisations
}

\author{
Stefania Boiano \\ Invisible Studio Ltd. \\ London \\ UK \\ stefania@invisiblestudio.net
}

\author{
Stefania Rossi \\ Museo Poldi \\ Pezzoli \\ Milan, Italy \\ s.rossi@poldipezzoli.it
}

\author{
Ann Borda \\ Health and Biomedical Informatics \\ The University of Melbourne \\ Australia \\ aborda@unimelb.edu.au
}

\author{
Guiliano Gaia \\ Invisible Studio Ltd. \\ London \\ UK \\ giuliano@invisiblestudio.it
}

\begin{abstract}
This paper explores how chatbots can offer opportunities for museums and galleries in engaging their audiences through recent developments, and through a case study approach focusing on the design and implementation of an audience development pilot in Milan involving four historic house museums (Case Museo di Milano). The pilot aimed to find new and interesting ways to engage teenagers in visiting these museums through visualising narrative using a convergence of chatbot technology and gamification.
\end{abstract}

Chatbots. Artificial intelligence. Museums. Gamification. Virtual museum guides. Teenagers.

\section{AN INTRODUCTION TO CHATBOTS}

Chatbots, also known as talkbots or chatterbots or bots, are computer programs that mimic conversation using auditory or textual methods. More specifically the functionality of chatbots use natural language processing (NLP) that has a history rooted in artificial intelligence (Al) (Corti et al. 2015, Dale 2017).

The concept of chatbots can be traced to Alan Turing's seminal publication Computing Machinery and Intelligence that addresses an overarching question: Can machines think? (Turing 1950, Dennett 2004, Hill et al., 2015, Copeland et al. 2017). The parameters of this question would shape the so-called Turing test. In its most simple form, the test is carried out as an imitation game. The test has a human interrogator speaking to a number of computers and humans through an interface. If the interrogator cannot distinguish between the computers and the humans then the Turing Test has been passed (Dennett 2004, Hill et al. 2015, Warwick 2017, Copeland et al. 2017).
One of the earliest such natural language applications was a chatbot called ELIZA developed from 1964 to 1966 at the MIT Computer Science and Artificial Intelligence Laboratory by computer scientist Joseph Weizenbaum (Weizenbaum 1966). ELIZA originally was created to use simple pattern matching and a template-based response (prewritten scripts) to emulate the conversational style of a Rogerian psychotherapist.

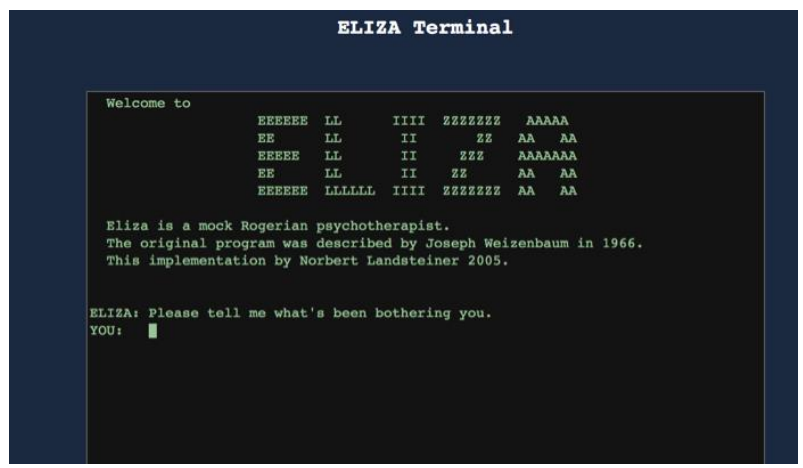

Figure 1: Image of ELIZA terminal interface accessed online (http://www.masswerk.at/elizabot/eliza.html). 
ELIZA generated global fascination in creating a natural language bot that might pass the Turing Test. The Loebner Prize launched in 1990 by Hugh Loebner in conjunction with the Cambridge Center for Behavioural Studies in Massachusetts (U.S.) is an example of a competition expressly designed to implement the Turing Test and builds on both the progressive interest and advances in $\mathrm{Al}$ developments (Loebner 2008).

Extending the pattern-matching techniques used in ELIZA and advancing NLP capabilities, American scientist Richard Wallace developed A.L.I.C.E. (Artificial Linguistic Internet Computer Entity) in the late 1990s. A.L.I.C.E., also known as Alicebot, is acknowledged for its pioneering programming using Artificial Intelligence Markup Language (AIML), which is an XML schema for creating natural language software agents. Wallace released the first version of AIML in July 2001, subsequently published the Pandora API on which A.L.I.C.E is based (Wallace 2008). A.L.I.C.E. became a three-time Loebner winner in 2000, 2001, and 2004.

Concurrent to A.L.I.C.E. developments, 'Jabberwacky' was being conceived by British programmer, Rollo Carpenter. Jabberwacky was intended to simulate "natural human chat in an interesting, entertaining and humorous manner" (Shah \& Warwick 2017). The emergence of the Internet provided Jabberwacky with a dynamic database of thousands of online human interactions from which to process responses. Jabberwacky under the guise of 'George' and 'Joan' won the Loebner Prize in 2005 and 2006 respectively.

In 2008, Jabberwacky launched a new iteration rebranded as 'Cleverbot'. Like Jabberwacky, Cleverbot is designed to learn from its conversations with humans (more than 150 million to date according to Wikipedia). It draws on past interactions to determine future questions and answers (Gehl 2014).

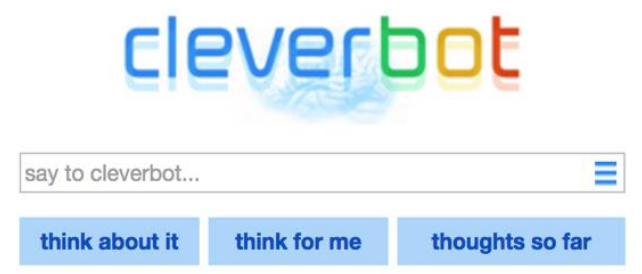

Figure 2: Cleverbot interface from Cleverbot website (http://www.cleverbot.com/).

In the endeavour to extend question-answering (QA) capabilities posed in natural language, IBM Watson was conceived in 2006 as a QA computing system with the goal of outperforming human contestants on the U.S. TV game show Jeopardy! IBM Watson was developed as part of IBM's DeepQA project (Ferrucci et al. 2010). Watson became the first computer to defeat contestants on the TV game show Jeopardy!, notably in a special match between Watson and Jeopardy! champions, Ken Jennings and Brad Rutter in 2011 (Best n.d.).

Chatbots in general are reaching milestones in artificial intelligence capability, as well as their pervasiveness in consumer facing products and services. For example in 2014, a chatbot called Eugene Goostman, portrayed as a 13-year-old Ukrainian boy, won an Al contest marking the 60th anniversary of Turing's death. In the Turing Test 2014 organised by the University of Reading, 33\% of the interrogators thought that Goostman was human (You 2015).

Created from AIML technology by programmer, Steve Worswick, 'Mitsuku' is a web-based chatbot available on the freeware instant messaging mobile app Kik Messenger and the Pandorabots website. It is among a growing number of sophisticated bots that can answer questions, play games, and is capable of basic reasoning in QA (Corti et al. 2015, Hill et al., 2015). Mitsuku is a three-time winner of the Loebner prize in 2013,2016 , and recently in 2017.

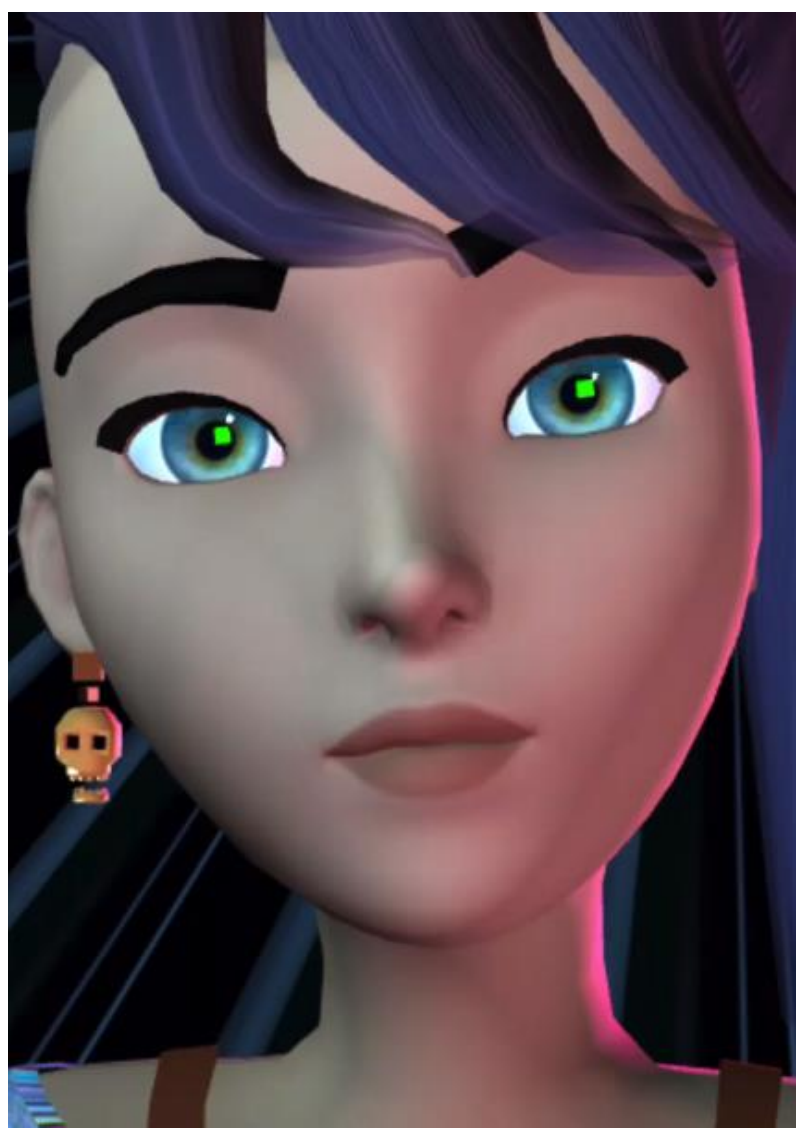

Figure 3: Mitsuku avatar from Pandora website (https://pandorabots.com/mitsuku/). 
Generally, the term 'chatbot' has referred to a software application that engages in a dialogue with a human using natural language. Most early advances have been associated with written language, but with advances in speech recognition, there is a narrowing of these associations. An early example is Dragon a simple speech understanding system developed in 1975 by Dr James Baker from Carnegie Mellon University. Other advances in the speech recognition sector have been made possible by VoiceXML, which has been published in a series of standards since the first release in the year 2000. The World Wide Web Consortium (W3C) has recently established in 2017 a W3C Community Group on Voice Interaction (http://www.w3.org/community/voiceinteraction),

which aims to explore the future of system-initiated directed dialogs of VoiceXML applications, such as voice assistants.

In just a couple of years, there has been an exponential rise of voice assistants such as Apple Siri launched in 2010, Google Now in 2012, Amazon's Alexa and Microsoft's Cortana in 2015, and Google Assistant in 2016. Using NLP and Internet of Things (IoT) platforms, these assistants connect to web services to answer questions and respond to user requests (Hoy 2018). Social media platforms are similarly incorporating chatbot functionality. Facebook opened up its Messenger platform and API to developers in 2017, providing a means to build a simple chatbot on Facebook. Twitter opened its direct messaging channel to chatbots in the previous year that began a hype cycle in twitterbots (Alarifi et al. 2016), and other messenger services are coming on board with open APIs (Hoy 2018, Mool 2018).

\section{MUSEUMS AND CHATBOTS}

Museums have been piloting related technologies of artificial intelligence and natural language processing as demonstrators for more than a decade (Boiano et al. 2003, Bickmore 2013, Swartout 2010, Borda \& Bowen 2017, Bordoni et al. 2016). Emerging free chatbot-creating platforms, e.g., Chatfuel, Chatterbot Eliza, among others, and the availability of open APIs, for instance, can offer both large and smaller museums the opportunity of experimenting with chatbots with relatively low effort while keeping costs and staff resources at a low level (Bordoni et al. 2016, Boiano et al. 2003) and potentially less impact on existing infrastructure (Dale 2016).

The exponential growth in the use of chatbots by marketers and online businesses in enhancing customer experiences, often as messaging applications that can personalise the interaction (e.g., recommender systems), is providing further comparable opportunities in the cultural sector (Swartout 2010). There are in fact a growing number of Museums currently going down this route, and using bots as part of their audience engagement programming.

For instance, the Heinz Nixdorf MuseumsForum in Paderborn Germany (http://www.hnf.de) has an early experience of using an avatar bot introduced as MAX. Developed in 2004, MAX is a conversational agent that directly engages with visitors through a screen as a virtual museum guide (Kopp et al. 2005)

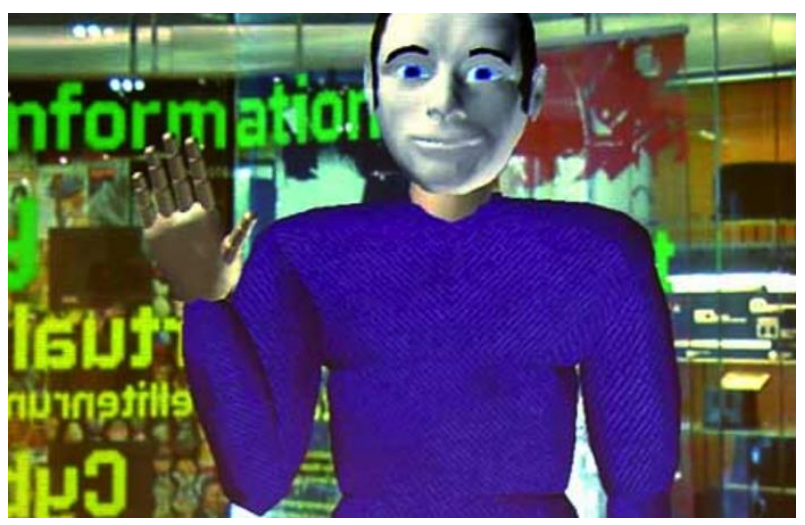

Figure 4: Avatar Max, Heinz Nixdorf MuseumsForum website, 2018 (https://tinyurl.com/yd3qd42q).

The Cooper-Hewitt Smithsonian Design Museum in New York City has been a pioneer in chatbot technologies with the creation of the Object Phone in 2013 in which a visitor can text or call to ask for more information about a museum object in the collection. In 2016, Object Phone became a subscription service so that a visitor can receive a daily update (http://objectphone.cooperhewitt.org).

In the words of Micah Walter, Director of Digital \& Emerging Media:

\begin{abstract}
I think institutions like museums have a great opportunity in the chatbot space. If anything it represents a new way to broaden our reach and connect with people on the platforms they are already using. What's more interesting to me is that chatbots themselves represent a way to interact with people that is by its very nature, bidirectional (Walter 2016).
\end{abstract}

The San Francisco Museum of Modern Art (SFMOMA) has developed Send Me SFMOMA (http://www.sfmoma.org/send-me-sfmoma), an SMS service that provides an approachable method of sharing the breadth of SFMOMA's collection with the public of which only $5 \%$ is seen in the Galleries at any one time (Mollica 2017). Using the service and texting the words "send me" followed by a keyword, a colour, or an emoji, a visitor will receive a related artwork image and caption via text message. 


\section{Hello?}

Hello from Send Me SFMOMA!

Text "send me cats" or "send

me of " to this number to

receive an artwork via SMS

from our collection.

Oh neat. Send me some

sunshine please.

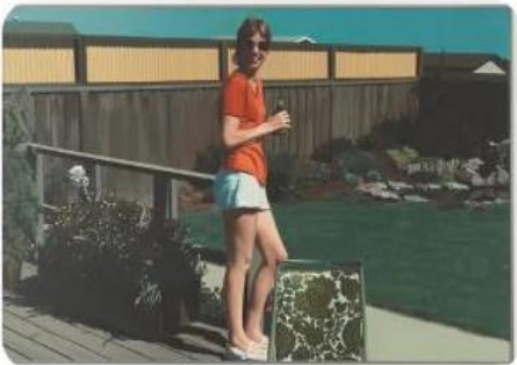

Robert Bechtle, 'Watsonville Olympia', 1977

Send me something pink

Figure 5: Image from Send Me SFMOMA blog. SFMOM, 2017 (https://www.sfmoma.org/read/send-me-sfmoma/).

In March 2017, the Anne Frank House museum (http://www.annefrank.org) in Amsterdam launched a Facebook Messenger chatbot that allows users to discover the personal history of Anne Frank and practical visitor information. Not simply a collections discovery bot, this application offers various conversation paths, allowing users to follow different paths in the Anne Frank story with concise information and links to additional content, for example, excerpts from her diary to the context of World War II at the time.

In 2017 the Museum of Australian Democracy in Canberra (http://www.moadoph.gov.au) marked the 50th anniversary of a landmark 1967 referendum in which Australians voted overwhelmingly to amend the Constitution to include Aboriginal people in the census and to allow the Commonwealth government to create laws for them. The Museum launched a referendum chatbot that allows visitors to learn about the historic and current impacts of this vote through chatting with it on Facebook Messenger. Directed towards children and accessible to adults, it uses simple gamification and responses, including emojis.

It acts like history in your pocket and is helping MoAD spark a conversation about the significance of the 1967 referendum. We're hoping it will be an effective way for people to get the facts, hear Indigenous perspectives on the referendum and reflect on its continuing relevance today. - Marni Pilgrim, Digital Engagement Manager, MOAD (O’Mallon 2017).

Not unlike the quick adoption of Facebook Messenger among some of the Museum examples, there is a rising trend in experimenting with Twitter bots, such as the Museumbot (@museumbot) that pulls open access images from a number of archives such as the Metropolitan Museum of Art. Other museum archive bots are steadily growing on Twitter, including the Los Angeles County Museum of Art LACMA bot (@LACMAbot), New York Public Library NYPL postcards bot (@NYPLpostcards), and the Museum of Modern Art MoMaR bot (@MoMARobot).

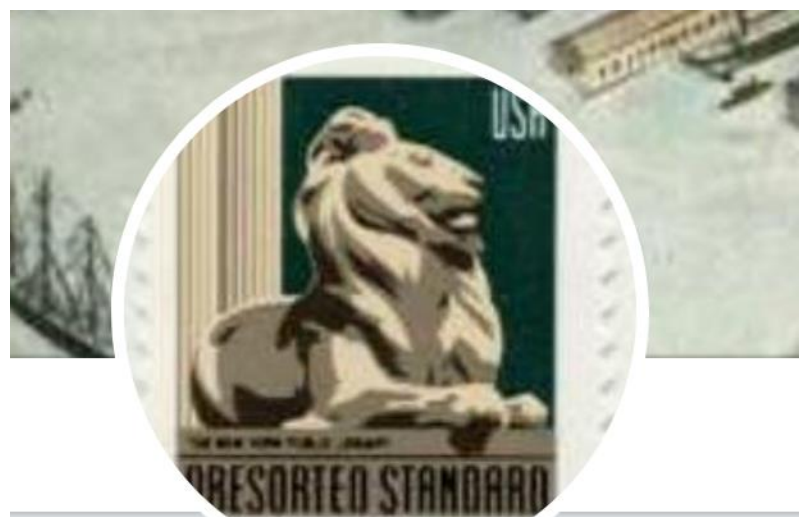

\section{NYPL Postcard Bot}

@NYPLpostcards

I am a bot that posts random postcards from the New York Public Library, four times a day. Bot by @backspace (Not affiliated with @NYPL)

○) New York, New York

圈 Joined January 2016

iv 3,170 Photos and videos

Figure 6: Image from NYPL Postcard Bot on Twitter (@NYPLpostcards) (retrieved 13 March 2018).

\section{HOUSE MUSEUMS OF MILAN: CASE STUDY}

The House Museums of Milan (Case Museo di Milano) is a group of 4 historical homes in Milan: Poldi Pezzoli Museum, Bagatti Valsecchi Museum, Necchi Campiglio Villa and Boschi Di Stefano House Museum (https://casemuseo.it). The House Museums launched a strategic initiative in 2016 
that aimed to motivate people to visit the four museums.

A London-based cultural innovation company, InvisibleStudio (http://www.invisiblestudio.it), was approached to introduce gamification into the engagement process, specifically to attract teenage audiences. The development of a chatbot-based game aimed at teenagers was a direction chosen by the House Museums curators and the InvisibleStudio team due to several factors.

Teenagers are a notoriously difficult public to engage in museums and often far more interested in social interactions between themselves than in cultural content - and this was proven to be even more challenging if that content is associated with a 19th century historic house. In general, teenagers visiting museums are identified with high levels of distraction and highly adapted to the use of social media (Kelly \& Russo 2008, Endo 2016, Fors 2016). Recent research in the U.S., for example, shows that online chatting is one of the highest forms of social interaction of teenagers (Statista 2017).

The InvisibleStudio team had already experimented with earlier chatbot technology in 2002, while working at the Museum of Science and Technology "Leonardo da Vinci" in Milan (Boiano et al. 2016, Boiano \& Gaiia 2017a). The process provided important lessons in the development of a chatbot application. This early chatbot was developed to mimic a Leonardo da Vinci character with whom the user would interact. This set high expectations for the user experience, and led to frustrations when the chatbot was not able to understand the user beyond simple introductory chat (Hill et al. 2015). Consequently, user issues occurred quite soon in the conversation (Boiano \& Gaia 2017a).

With these lessons in hand, InvisibleStudio changed their approach when creating a chatbot for the House Museums project. The chatbot would only be used as a tool or "virtual companion" to help younger audiences solve a game set in the real physical environment of the museum. By engaging teenagers through gamification, it could shift the user's focus from the conversation with the chatbot to the actual exploration of the Museum collections (Boiano 2016, Cawston 2017).

The chatbot game for the House Museums was developed using Facebook Messenger, and intended mainly for young users and teenagers to engage them in exploring the four homes. Aspects of visualisation and storytelling were utilised with the chatbot platform to simulate a teenaged girl asking visitors to help her in defeating a mysterious Renaissance magician (based on a real historical figure) by solving mysteries in the galleries.
Exploration is encouraged by users in looking for hidden clues that lead to a final discovery, which provides a further incentive to engage with the application.

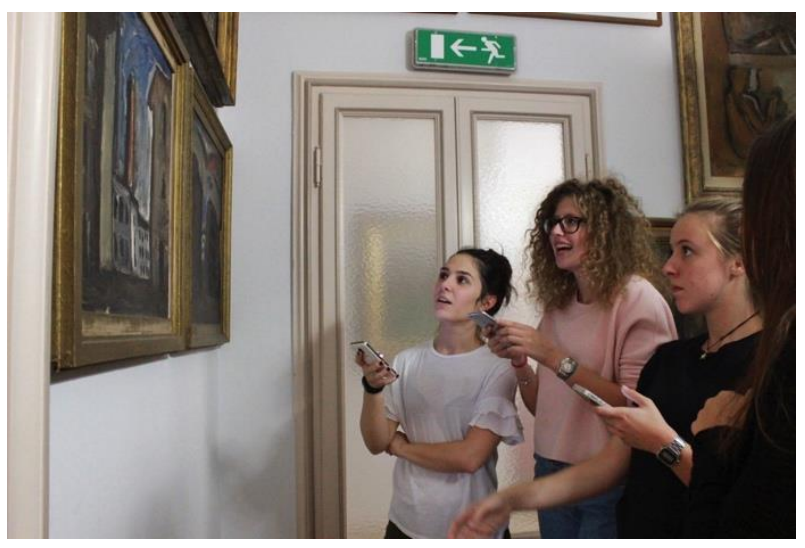

Figure 7: Teenagers using the chatbot game in Milan. Source: InvisibleStudio, 2017.

Before operational release of the chatbot, it was piloted with teenagers aged 16-18. The pilot was conducted by InvisibleStudio with 80 teenaged students from local high schools in Milan. The pilot resulted in the following findings:

- $90 \%$ of students managed to complete the game

- $30 \%$ had connection problems

- $34 \%$ were worried for their data traffic

- $88 \%$ found the length of the game was right

- $72 \%$ evaluated the game as highly entertaining

- $66 \%$ found it a useful learning tool, especially if it was used with another student or in a small group.

These results offered some clear directions for the final development stages. Especially interesting for the developers was the fact that students liked using the chatbot in small groups, rather than on their own, because the game triggered collaboration within the team and created a friendly competitive environment with other teams. (Boiano \& Gaia 2017b)

At the same time, the pace and quality of the conversation emerged as a critical aspect for future consideration, requiring a larger effort from the development team to create engaging and realistic non-linear narratives (Hill et al. 2015). For instance, key features which required considerable tweaking before publishing the application included: making conversations more realistic by studying real chats between users and guides, referencing objects which the user can actually see "here and now" in the galleries, and finding the perfect length for the game (Boiano \& Gaia 2017a, 2017b). 
Another key challenge was the necessity of keeping open a continuous online connection between users and the chatbot. This can prove difficult in historical house museums, where the older infrastructure is comprised of complex layouts and thick walls, which can prevent wireless connectivity or an even distribution of Wi-Fi connections.

Other challenges still need to be addressed. These are mainly related to the Facebook Messenger platform itself, and changing preferences by teenagers and audiences for different messaging services and platforms which may not have open APIs for chatbot development at this stage, such as WhatsApp (Mool 2018). With the possibility of more open APIs, museum chatbots could be developed for a potentially larger audience (e.g., teenagers), and potentially a larger uptake, which does not depend on a subscription.

Despite the current limitations, the advanced chatbot application in the House Museums has become successfully operational through design thinking, design iteration, and importantly, teenagers' participation in the development process.

\section{CONCLUSION}

There is clear evidence of the potential of cultural heritage organisations to play a significant role in the development of chatbots. The sector already has a strong provenance with decades long experience in piloting emerging and embedded technologies relevant to smart city services, and in its understanding of user-centric interaction characterised by context-awareness, personalisation, and adaptation.

What emerged from this project is that the convergence of chatbot and gamification has proved to be a powerful tool in involving younger, digital savvy generations in novel and interesting ways to them (Cawston et al. 2017, Kelly \& Russo 2008, Endo 2016, Fors 2016).

Our findings particularly suggest that users enjoy interacting with a chatbot in a game context, and that this engagement can provide a smarter way of leading younger audiences to interact with objects and historic environments with greater attention. However with all the successes of this chatbot launch, there also remain challenges that need further consideration beyond the scope of this paper. As mentioned above, the availability of a wider range of chatbot platforms is one such challenge. A more involved issue is the pace and quality of the bot conversation which emerged as a critical aspect of this project. The present chatbot application required a bigger effort from the developing team to create engaging and realistic non-linear narrative lines, and this will be part of a continuing iteration in future developments.

\section{REFERENCES}

Alarifi, A., Alsaleh, M., and Al-Salman, A. (2016) Twitter Turing Test: Identifying social machines. Information Sciences, 372, 332-346.

Best, J. (n.d.) IBM Watson: The inside story of how the Jeopardy-winning supercomputer was born, and what it wants to do next. TechRepublic. https://www.techrepublic.com/article/ibm-watsonthe-inside-story-of-how-the-jeopardy-winningsupercomputer-was-born-and-what-it-wants-to-donext/ (retrieved 13 March 2018).

Bickmore, T. W., Vardoulakis, L. M. P., and Schulman, D. (2013) Tinker: A relational agent museum guide. Autonomous agents and multiagent systems, 27(2), 254-276.

Boiano, S., Cuomo, P., and Gaia, G. (2016) Realtime Messaging Platforms for Storytelling and Gamification in Museums: A case history in Milan. In Proceedings Electronic Visualisation and the Arts (EVA London 2016), BCS, London, 12-14 July 2016. DOI: 10.14236/ewic/EVA2016.60

Boiano, S., Gaia, G,. and Caldarini, M. (2003) Make Your Museum Talk: Natural Language Interfaces for Cultural Institutions. Museums and the Web 2003.

https://www.museumsandtheweb.com/mw2003/pap ers/gaia/gaia.html (retrieved 13 March 2018).

Boiano, S. and Gaia, G. (2017a) 3 Lessons learnt from Building our first Museum Chatbot... 15 years ago! Invisible Studio, 13 June 2017.

https://medium.com/@invisiblestudio/3-lessons-

learnt-from-building-our-first-museum-chatbot-15years-ago-7189c8a8fe6 (retrieved 13 March 2018).

Boiano, S. and Gaia, G. (2017b) 5 Tips for Involving Teenagers in Your Museum Using a Chatbot. Invisible Studio, 27 June 2017. https://medium.com/@invisiblestudio/5-tips-forinvolving-teenagers-in-your-museum-using-achatbot-bf88ff3ad568 (retrieved 13 March 2018).

Borda, A. and Bowen, J. (2017) Smart Cities and Cultural Heritage: A Review of Developments and Future Opportunities. In Proceedings Electronic Visualisation and the Arts (EVA London 2017), BCS, London, pp.9-18.

Bordoni, L., Mele, F., and Sorgente, A. (eds) (2016) Artificial Intelligence for Cultural Heritage. Newcastle: Cambridge Scholars.

Cawston, R., Efergan, D., and Green, L. (2017) It's in the game: can playful digital experiences help organisations connect with audiences in new 
ways? Museums and the Web: MW 2017, January 31, 2017. https://mw17.mwconf.org/paper/its-in-thegame-can-playful-digital-experiences-helporganisations-connect-with-audiences-in-newways/ (retrieved 13 March 2018).

Copeland, J., Bowen, J. P., Sprevak, M., Wilson, R., et al. (2017) The Turing Guide. Oxford University Press.

Corti, K. and Gillespie, A. (2015) A Truly Human Interface: Interacting face-to-face with someone whose words are determined by a computer program. Frontiers in Psychology, 18 May 2015. DOI: 10.3389/fpsyg.2015.00634

Dale, R. (2016) The Return of the Chatbots. Natural Language Engineering, 22(5), 811-817. DOI: $10.1017 / S 1351324916000243$

Dennett, D. (2004) Can Machines Think? In Alan Turing: Life and Legacy of a Great Thinker, ed. C. Teuscher, 295-316, Berlin: Springer. DOI: http://doi.org/10.1007/978-3-662-05642-4 12

Endo, T. (2016) Teens Use Tech to Talk Art: Amplifying teen voice and art interpretation. MW2016: Museums and the Web 2016, March 10. http://mw2016.museumsandtheweb.com/paper/tee ns-use-tech-to-talk-art-amplifying-teen-voice-andart-interpretation/ (retrieved 13 March 2018).

Ferrucci, D., et al. (2010) Building Watson: An overview of the DeepQA project. Al Magazine, 36(3), 59-79.

Fors, V. (2016) Teenagers' Multisensory Routes for Learning in the Museum. The Senses and Society, 8(3), 2016, 268-289. DOI:

$\underline{10.2752 / 174589313 \times 13712175020479}$

Gehl, R. W. (2014) Teaching to the Turing Test with Cleverbot. Transformations: The Journal of Inclusive Scholarship and Pedagogy, 24(1-2), 5666.

Hill, J., Ford, W. R., and Farreras, I. G. (2015) Real conversations with artificial intelligence: $A$ comparison between human-human online conversations and human-chatbot conversations. Computers in Human Behavior, 49, 245-250.

Hoy, M.B. (2018) Alexa, Siri, Cortana, and More: An Introduction to Voice Assistants, Medical Reference Services Quarterly, 37(1), 81-88.

Kelly, L. and Russo, A. (2008) From Ladders of Participation to Networks of Participation: Social Media and the Museum Audiences. MW2008: Museum and the Web Conference, April 9-12, Montreal, Canada.

Kopp, S., Gesellensetter, L., Krämer, N. C., and Wachsmuth, I. (2005) A conversational agent as museum guide-design and evaluation of a realworld application. In International Workshop on
Intelligent Virtual Agents, pp.329-343. Springer, Berlin, Heidelberg

Loebner, H. (2008) How to Hold a Turing Test contest. In: R. Epstein, G. Roberts, and G. Beber (eds) Parsing the Turing Test: Philosophical and Methodological Issues in the Quest for the Thinking Computer, 173-179, New York, Springer. DOI: 10.1007/978-1-4020-6710-5 12

Metz, R. (2017) Three Weeks with a Chatbot and I've Made a New Friend. MIT Technology Review March 23, 2017.

https://www.technologyreview.com/s/603936/threeweeks-with-a-chatbot-and-ive-made-a-new-friend (retrieved 13 March 2018).

Mollica, J. (2017) Send Me SFMOMA. SFMOMA, July. $\quad$ https://www.sfmoma.org/read/send-mesfmoma/ (retrieved 13 March 2018).

Mool, T. (2018) Chatbot Trends: The Year of the VoiceBot, WhatsApp Bots, MaaP. NativeMSG. https://nativemsg.com/blog/2018-chatbot-trendsthe-year-of-the-voicebot-whatsapp-bots-maap (retrieved 13 March 2018).

O'Mallon, F. (2017) Museum of Australian Democracy uses Facebook Messenger chatbot to connect with Australia's past, Canberra Times May 26, 2017. http://www.canberratimes.com.au/actnews/museum-of-australia-democracy-usesfacebook-messenger-chatbot-to-connect-withaustralias-past-20170525-gwcvu5.html (retrieved 13 March 2018).

Robinson, S., Traum, D. R., Ittycheriah, M., and Henderer, J. (2008) What Would You Ask a Conversational Agent? Observations of HumanAgent Dialogues in a Museum Setting. In Proceedings Language Resources and Evaluation Conference, Marrakech, May 2008. (retrieved 13 March 2018).

Shah, H., and Warwick, K. (2017) Machine humour: examples from Turing test experiments. Al \& Soc 32, 553-561. DOI: $10.1007 / \mathrm{s} 00146-016-0669-0$

Statista (2017) Reach of leading social media and networking sites used by teenagers and young adults in the United States as of February 2017. https://www.statista.com/statistics/199242/socialmedia-and-networking-sites-used-by-us-teenagers/ (retrieved 13 March 2018).

Swartout, W., et al. (2010) Ada and Grace: Toward realistic and engaging virtual museum guides. In International Conference on Intelligent Virtual Agents, pp.286-300. Springer, Berlin, Heidelberg.

Turing, A. M. (1950) Computing Machinery and Intelligence. Mind, 49, 433-460.

Wallace, R. (2008) The Anatomy of A.L.I.C.E. In R. Epstein, G. Roberts, and G. Beber (eds.) Parsing the Turing Test: Philosophical and Methodological 
Issues in the Quest for the Thinking Computer, 181-210, New York, Springer). DOI: 10.1007/9781-4020-6710-5 13

Walter, M. (2016) Object Phone: The continued evolution of a little chatbot. Cooper Hewitt Labs, July 4, 2016.

https://labs.cooperhewitt.org/2016/object-phonethe-continued-evolution-of-a-little-chatbot/ (retrieved 13 March 2018).
Warwick, K. and Shah, H. (2016) The Importance of a Human Viewpoint on Computer Natural Language Capabilities: A Turing test perspective. Al \& Soc 31(2), 207-221.

Weizenbaum, J. (1966) ELIZA-a computer program for the study of natural language communication between man and machine. Communications of the ACM, 9(1), 36-45.

You, J. (2015) Beyond the Turing Test. Science 347, 116. DOI: $10.1126 /$ science.347.6218.116 